\title{
Lingua-pragmatic potential of speech actions in medical professional discussions
}

\author{
N. L. Shamne \\ German and Roman philology department \\ VolSU \\ Volgograd, Russia \\ nikolay.shamne@volsu.ru
}

\author{
M. S. Nevzorova \\ German and Roman philology department \\ Volsu \\ Volgograd, Russia \\ nevzorovamaria@gmail.com
}

\begin{abstract}
This paper examines medical professional communication that has not been studied extensively: medical professional discussions on current medical issues. The research is based on English texts of medical scientific discussions, freely available on the Internet. The novelty of the research lies in the fact that medical professional communication qualifies as equal status communication because it is a collaboration of specialists. Medical professional communicative behavior is considered in terms of the system of speech actions verbalized by means of linguistic devices and governed by conventionally accepted norms. Four types of speech actions are identified: connective speech actions directed to establishing contact among peers, metacommunicative speech actions regulating the communicative process, directive speech actions aimed at development of the discussion, cognitive speech actions serving for sharing professional knowledge and expressing expert's viewpoints. The language of medical professional discussion has significant consequences for those who use it. Participants tend to mitigate categorical assertions and support positive atmosphere using a variety of linguistic and rhetorical devices.
\end{abstract}

Keywords-medical discourse, communicative behaviour, speech action, medical professional discussion.

\section{INTRODUCTION}

This paper examines a significant area of medical professional communication: scientific professional discussion by medical experts, physicians. Although doctor - patient communication is in the focus of linguists' attention, little is known about doctor - doctor interaction. Interaction in a medical field is not limited by doctor -patient communication, doctors talk to each other about patients, methods of treatment, diagnostics, medical procedures; they share medical knowledge among specialists increasing their professional competence during daily rounds, scientific conference, round table discussions, medical videoconferences, etc. Effective communication requires building communicative behavior according to particular strategies and tactics to collaborate with different medical specialists. By analyzing the communicative actions of professional participants in medical professional discussions, a lot of can be learned about medical professional discourse.

\section{THE STUDY OF MEDICAL DISCOURSE}

The aim of the article is to reveal the system of communicative actions composing a communicative behavior of medical specialists during medical scientific professional discussion. The analysis of medical professional discussion is based on the study of scientific professional discussion and the study of medical discourse. Linguists have devoted more attention to the study of interactions occurring between doctors and patients. The findings of studies suggest that the doctor-patient interaction is asymmetrical, so a physician plays a crucial role in development of the communicative process [1-4]. There is a significant omission in very extensive literature on medical discourse because very little is written about intraprofessional interaction. There are few research works devoted to different aspects of medical professional communication. Close relationship between written and oral communication in clinical practice is considered as specific characteristic of medical professional communication, as case presentations, produced by physicians, refer to written forms (patients' case histories, clinical notes) [5]. Written forms of medical professional discourse is investigated by Pettinari in terms of how surgeons make notation about performed operations revealing objective laws for transferring professional knowledge into the professionally restricted textual form [6]. The study of formal case histories presentation made by interns or residents shows that case presentation serves as a vehicle for professional socialization [7]. All these works are attempts to prove a significance of medical professional interaction investigation for understanding the complexity of contemporary medical discourse.

\section{COMMUNICATIVE BEHAVIOUR AND SPEECH ACTIONS}

The study is based on the assumption that communicative behavior is behavior (verbal or nonverbal) of a person or a group in the communicative process governed by norms and traditions accepted in that community [8]. Communicative behavior is a combination of speech actions verbalized by means of linguistic devices. Professional communication in the medical sphere is held as a dialogue [9], composed of elementary speech actions. Their distinctive characteristic is speaker's intentions. The key intentions of professional communication, as Skorikova proves, are divided into 
communicative and communicative-notional. The former is directed to the organization of communicative acts, while the latter is directed to connect communicative partners. Communicative intentions include stimulation for communication, a request to give a definition, an expression of agreement or disagreement, an opinion support. Topic or issue announcement, objectivation, verification, explanation, conclusion are referred to communicative-notional intentions. It is pointed out that communicative intentions appear in the dialogue, while communicative-notional intentions exhibit in the monologue as it is less complicated with linguistic functions (communication and persuasion) than the dialogue is.

This research work is based on the analysis of texts of medical professional discussions freely available on the Internet. More than 30 discussions were examined. As the analysis has shown, all medical professional discussions tend to follow an almost ritualized format, characterized by usage of certain polite phrases, syntactic forms and specific organization. The medical professional discussion is a speech event which serves both to impart information and as a vehicle for production of new professional knowledge among peers [10]. This speech event has a symmetrical communicative mood, as participants are equal in virtue of having medical education, performing similar institutional functions, possessing shared thesaurus (medical terms, medical slang). There are at least three main phases in the structure of event: the introduction, the main part and the conclusion. Specific speech actions are realized at each stage of the discussion. At the first stage, speech actions are used to connect participants, to announce the topic and goals of the following discussion and to introduce all experts. The discussion usually starts with the presentation of the participants and the moderator, but there are exceptions when the participants introduce themselves. Although the discussion is a dialogue, the first stage of this communicative event is usually held as a monologue of a moderator. The moderator starts off the conversation by welcoming his colleagues to take part in the discussion, poses a question and asks each participant to comment briefly on the question, then asks follow-up questions to clarify points or foster discussion.

The main part of the discussion is a dialogue represented in the form of question - answer. Answering the question, an expert may express his or her ideas rather at length, so it can appear to be a monologue. Speech actions of this stage can be divided into two groups. The first group includes actions that regulate a discussion progress: to ask for an expert opinion, to challenge sharing ideas, to clarify the information, to announce a new theme or subtheme, to impart additional information, to support an opinion, to agree (or disagree) with a participant(s). Actions of the second group are meant to organize logical and conceptual framework of the utterance: to support viewpoints with arguments.

The aim of the final stage is to conclude a discussion. Speech actions at this stage are directed to evaluate the discussion, to summarize main ideas, to express an overall decision and to thank all participants for coming.
The finding shows that speech actions of medical professional discussion can be classified according to the underlying intention. The table "Fig. 1." below shows the correspondence of the speech action with its type in the medical professional discussion.

\section{CONNECTIVE SPEECH ACTIONS}

The communicative behavior of the first stage of medical professional discussion is composed of connective speech actions aimed at interconnecting of the participants. As Sidorova states, connective speech actions include greetings, establishing connection, vocatives, and finishing the conversation. Such speech action is distinguished from functionally similar metacommunicative action on the ground that the latter regulates communicative activity of the participants [3]. The connective speech actions are externalized by means of polite expressions:

K.1: Good afternoon. My name is Andrew D., and I am Professor of Clinical Geriatrics at the University of Alabama at Birmingham.

This conversational turn includes not only greeting but also self presentation. At the first stage of a medical professional discussion such speech actions are formal and to a certain extent ritual. Greeting is also expressed by hello or good afternoon. These words are stylistically formal, so using them is intended to emphasize speaker's attitude toward an official character of the following conversation.

Formal invitations to join in the discussion may function as connective speech actions, for example:

K.1: Let me welcome you all to the Expert Medical Roundtable on high-density lipoprotein $(H D L)$ cholesterol and triglycerides in the year 2011.

Here the moderator uses a polite expression to greet all members of the discussion. In examined medical professional discussions, the invitation to join in the conversation can be verbalized by means of polite phrases: Welcome to this roundtable discussion; It's my pleasure to welcome everyone to this roundtable, etc.

Presentation (self-presentation) of the participants is obligatory in the medical professional discussion. The frequency of use and compulsory character of this speech action in examined discussions allow defining it as typical and even ritual for medical professional communication.

TABLE I. SPEECH ACTIONS IN MEDICAL PROFESSIONAL DISCUSSION.

\begin{tabular}{|l|l|}
\hline \multicolumn{1}{|c|}{$\begin{array}{c}\text { A type of a speech } \\
\text { action }\end{array}$} & \multicolumn{1}{c|}{ A speech action } \\
\hline $\begin{array}{l}\text { Connective speech } \\
\text { actions }\end{array}$ & $\begin{array}{l}\text { Establishing contact } \\
\text { finishing contact } \\
\text { presentation (self presentation) of the participants } \\
\text { announcement of the topic. }\end{array}$ \\
\hline $\begin{array}{l}\text { Metacommunicative } \\
\text { speech actions }\end{array}$ & $\begin{array}{l}\text { Joining in discussion signals } \\
\text { attracting signals } \\
\text { supporting signals. }\end{array}$ \\
\hline Directive speech actions & $\begin{array}{l}\text { Requests } \\
\text { offers } \\
\text { challenges. }\end{array}$ \\
\hline Cognitive speech actions & $\begin{array}{l}\text { Explicating a viewpoint } \\
\text { presenting arguments } \\
\text { expressing agreement or disagreement. }\end{array}$ \\
\hline
\end{tabular}


Presentation (self-presentation) becomes significant for introduction of participants, and it implicitly states a high professional competence of the invited medical experts; it also helps to establish positive attitude for the following communication amongst peers.

Establishing contact with participants is performed by means of polite frames which serve as a ritual to greet and to outline roles in the following discussion.

K.1: I am honored to moderate today's discussion with very well recognized experts in hypertension: true "doctor's doctors", if you will. Our panelists include Dr. J. from South Carolina, Dr. A. from Pittsburgh, and Dr. M. from New York. Welcome, gentlemen.

The opening frame I am honored to moderate today's discussion... serves on the one hand as polite expression, but on the other hand influences participants' positive attitude. In the medical professional discussion such frames are also represented by:

- It's my pleasure...

- I'm really grateful to all three of you...

- We are privileged to have three expert clinicianscientists with us...

- It's my privilege ....

Besides this, the utterance includes positive evaluation ("doctor's doctors") of a high level competence and professionalism of the invited experts. Emotional effect is intensified using very well and true. This technique aids to establish an atmosphere of cooperation and collaboration.

Universal means of establishing contact is vocative. According to its nature vocative is multifunctional. In medical professional discourse vocatives have two functions: phatic (to name the next speaker) and appellative (to appeal to a participant as an intelligent colleague and a highly qualified specialist).

Specific feature of medical professional communication is primarily formal communication, as all participants act in accordance with their role in this communicative event (a moderator - an invited expert). Communicative role imposes a subject of communicative process to meet social expectations, thus participants tend to adhere to social distance. Vocatives serve as official markers. In medical professional communication a prototypical form is vocative composed of special vocative $D r$. (Doctor) and last name (Dr. D., let me start with you. Doctor B., let me ask you the next question). These vocatives mark a high status of an addressee. If communicative partners are equal in the communicative status, it is possible to use only first name instead of special vocative to make a situation less formal. The analysis of medical professional discussions shows minimizing the distance between the participants using only first name to address a communicative partner (Let me start with a question for Dr. B.; Brad, can you give us your analysis of this trial?).

At the final stage, speech actions concluding the discussion are used. It is important to summarize the views and ideas, to draw conclusion and to thank all participants for their contributions. For purpose the following phrases are used:
Thank you, everyone. I really appreciate your participation and very insightful comments. Thank you all very much for your valuable insights.

Thus, connective speech actions play a significant role in developing medical professional discussion as they aid to establish contact between participants, to harmonize the communicative process, and to conclude discussion effectively. These actions should be distinguished from metacommunicative speech actions, which are defined in the next section.

\section{MetacommunicAtive SPEECH ACtions}

Metacommunication is defined as communication about communication. Communication is primarily an information exchange, but to make this process effective special signals (markers) are used. These signals are aimed to provide reliable communication. Metacommunicative speech actions may perform cognitive function (to promote understanding between participants) and regulative function (to organize or to correct a communicative process).

The analysis of medical professional discussions allows eliciting such speech actions as joining in discussion signals, attracting signals, supporting signals.

Join in discussion signals are represented by metacommunicative commentary. Their specific characteristic is that they are aimed to connect moderator's initiative move and expert's reactive move. Here is an adjacency pair from medical professional discussion. Situational context: A. - a moderator, B. - an invited expert.

A.: Dr. T., what is the difference between the terms diastolic dysfunction and diastolic heart failure? Is there an important difference?

B.: I would like to echo a couple of points that Dr. D. stated. The reason why we need to discuss diastolic function on every echocardiogram is that diastolic dysfunction (abnormal filling/relaxation) is present in virtually all patients with heart failure.

Metacommunicative commentary I would like to echo a couple of points that Dr. D. stated includes a reference to the previous speaker represented indirectly by means of would like and, thus is more polite than direct reference. Modal modifier would like coupled with the verb infinitive with semantic "express an opinion" (say, add, follow, raise, make comment) aids to convey implicitly a doubt in desirability of this action for listeners that is considered polite for English culture [11]. Less polite but conventionally acceptable are phrases: I want to respond to part of your question, with respect to required expertise and understanding of the systems. Let me bring in this perspective that you've heard me talk about. To Bill's point about weight loss...

Attracting signals are expressed by requests to ask a question or to make a comment. Here is a fragment of the discussion. Situational context: A., B. - invited experts, C. - a moderator. 
A.: Didn't Pun show in a review of the DaVita database that, yes, a low potassium bath was associated with increased cardiovascular mortality, but so was a low calcium bath?

B.: Yes. They both potentiate each other. So, the low potassium, the low calcium, the low magnesium, and the high bicarbonate all are impacting the $Q T$ interval and are all arrhythmogenic by their nature.

\section{C.: Let me ask a question to both of you before we hear} Pieter's thoughts. ...

Medical professional discussion as a form of formal official communication is governed by a set of rules and conventionally accepted norms of communicative behavior. Participants have to comply with these rules to communicate effectively [12]. According to the Organization of TurnTaking for Conversation, a new speaker may begin at transitional-relevant point when the previous speaker ends. So the moderator may intervene in the conversation when the participant B. gives a particular signal So for pragmatic completeness of the utterance. The moderator formally asks for permission to intervene, but functionally this utterance serves as an attracting signal, because the right to ask questions in the discussion process belongs to a moderator. It is a kind of convention. Attracting signal is expressed in polite form Let me ask a question... . Illocutionary point of the utterance makes a speaker dependent from his partner; therefore it aids to minimize the imposition on the participant and to avoid a communication failure. Among these attracting signals are Let me ask a question to both of you before we hear P's thoughts. Let me just briefly comment.... Let me make one quick comment.

Supporting signals are represented by lexical markers of agreement (yes, sure, okay), etiquette phrases to say thanks (thanks, thank you), less informative particles (well, ah). Such signals are aimed to support the development of communication.

\section{DIRECTIVE SPEECH ACTIONS}

Directive speech actions in the medical professional discussion connect with impact on communicative partners to provide some sort of information: to comment, to express their viewpoints, to describe special procedures, etc. Directive speech actions are aimed to stimulate partners' communicative activities and may be expressed by variety of ways: questions, requests, offers, challenges. In English culture directives are considered as Face Threatening Acts since may restrict personal freedom of the communicative partner. It is accepted to distinguish face-threatening acts into: those predicating a future action of the hearer (orders, requests) and those predicating a future action of the speaker toward the hearer (offers). According to Brown and Levinson's theory, such actions are required to mitigate their potential face-threats. As Larine states, directive speech actions have to be expressed indirectly.

Communication process in the medical professional discussion is built on question - answer sequences. Asking a question seems to impose on the communicative partner, committing him to answer, and so this speech action is considered as face-threatening [13]. The analysis of medical professional discussion shows that it is conventionally accepted to mitigate or soften directive speech actions of asking a question by using a hedge or state a question indirectly. In English culture the most extended mitigating device is "the question-hedged strategy where one reformulates a direct request or order as a question, thereby producing an indirect request" [13]. Consider the fragment of medical professional discussion, where the moderator (A.) asks question to elicit information from the expert. To soften the face-threatening aspect of asking a question he reformulates it into an indirect request.

A.: Dr. W., regarding the informed decision-making process, ACS has consistently supported this concept. Can you define informed decision making and explain its importance?

B.: In general, whenever there is an intervention or a diagnostic tool where the balance between the benefits and potential harms is too close to call, it is really incumbent on physicians to engage patients in an informed decision as to whether they wish to proceed....

Indirect polite requests are the main form of challenging communicative partners to share ideas and viewpoints as it is conventionally accepted in medical professional discussions. There is a variety of hedges for mitigating such requests. Here are some examples of mitigating by means of modals:

- Can you describe the ALARM-HF project a bit?

- Can you summarize the USPSTF recomendation and explain why you

- Would you like to begin with your theories on vasodilation in $H F$ ?

Although the first two utterances are presented in a form of information question, but the last one is suggestion/offer/request question, these utterances are formal questions, aimed at eliciting a participant's answer. The straightforwardness of the utterance may be decreased by indirect question: Specifically, I'd like to have some comments about device approval... The speaker avoids challenging his partner directly, using I'd like aids in expressing doubting in the hearer's possibility to perform this action. Thus the moderator implicates that he respect culturally determined and conventionally accepted politeness norms.

The role of mitigating device can be played by modal modifier minimizing the imposition, like just, a bit, a little. Let us consider the following examples from examined medical professional discussions:

- Can I ask you, Jon, to talk a little bit more or expand a little bit more about...

\section{- Can you expand on that a little bit?}

These utterances combine indirect question with modal modifier minimizing the imposition to soften face-threatening aspect of eliciting information. The more mitigating devices are used in the utterance, the more indirect and so the more polite it sounds. 
The analysis of medical professional discussion shows that it is possible to use a soften imperative making a communicative partner provide an answer:

\section{B., please discuss dalecetrapib and the clinical trial... \\ B., please explain the differences between an inhibitor...}

Here mitigation is performed by means of the word please.

Directive speech actions may be expressed in an offer form. Let us consider the fragment of medical professional discussion, where the moderator (A.) asks to share partner's viewpoint (B.):

A.: Dr. D., let's examine diabetic compared to nondiabetic patients. What type of clinical trial data do we have for the role of therapy of high triglycerides and low HDL levels and the difference in diabetic and non-diabetic populations?

B.: Unfortunately, we don't have a lot of evidence to focus on in the studies that compare one to the other...

The phrase let's expresses formally an offer, but the illocutionary point of this utterance is a request to elicit information.

Thus, directive speech actions may be represented in a variety of ways: indirect requests, indirect questions, modal modifiers minimizing the imposition. It is conventionally accepted in medical professional communication to express direct speech actions indirectly to soften them and make them more polite.

\section{COGNITIVE SPEECH ACTIONS}

Cognitive speech actions are considered as actions aimed at presenting arguments as a proof or evidence for a fact, making references to different sources, clarifying and explaining a viewpoint regarding a discussed question, expressing agreement or disagreement with partner's position. This type of actions is intended to clarify and prove a speaker's propositions. These speech actions primarily present a communicative line of discussion to participants rather than a communicative line to a moderator, but there are exceptions if a moderator takes part in negotiations as a participant.

The development of medical professional discussion is built as a viewpoint exchange, and one should prove to have his or her ideas accepted by other participants. A course of reasoning aimed at demonstrating a truth or falsehood of a fact have to be organized logically to affect communicative partners. In the medical professional discussion a participant usually answering a moderator's question provides a sequence of cognitive speech actions which is composed of a proposition (that is maintained by arguments), arguments (a set of statements), a fact or an example (those illustrating a proposition or arguments). It may be added with conclusion, evaluation and counterargument.

In fact, the viewpoint exchange retains features of a scientific article (precision, a high occurrence of medical terminology, clear and scientific style), but presence of the communicative partners leads to using more expressions of uncertainty [14]. As medical academic writing, the medical professional discussion is highly hedged. Hedging in medical discourse is multifunctional: "help protect the writer against possible wrong interpretations or faulty results, but they also allow them to demonstrate an awareness of the reader's possible alternative viewpoint, displaying the conditional nature of statements out of strategic respect for them and indicating the degree of confidence that the writer judges it prudent to attribute to statements" [15].

The analysis shows that medical professional discussion is an argumentative and persuasive type of communication. Participants expressing their viewpoints produce interesting, novel and plausible professional knowledge; thus, trying to avoid personal responsibility for statements and to leave the opportunity for hearer's alternative view. Let us consider a fragment of a discussion:

A.: ... What is new in echocardiography for diastole evaluation in terms of evaluation of strain?

B.: Myocardial strain assessment does provide additional information in the assessment of systolic and diastolic function. Strain imaging can uncover systolic impairment that may not be apparent by simply looking at the EF. Furthermore, it may also be helpful in assessing diastolic function to some extent, since tissue Doppler imaging is limited by a couple of factors. First, it has the angledependency problem, which leads to underestimation of the measured velocities. Second, tissue Doppler may be erroneously normal due to the translational motion of the heart. These 2 factors may lead to errors in the tissue Doppler assessment of diastolic function. Strain imaging, on the other hand, implements speckle-tracking technique, rather than Doppler, to evaluate the displacement of 2 echo speckles within the myocardium relative to each other. Therefore, it is not affected by the angle-dependency problem or the translational motion of the heart. Strain imaging, however, is not widely used clinically in the assessment of diastolic impairment.

Here the expert's contribution to the conversation is rather long and may be related to a monologue. The communicative partner tries to share his viewpoint with participants so his explanation is built on a sequence of arguments proving the main proposition "Myocardial strain assessment does provide additional information in the assessment of systolic and diastolic function" stating advantages of a new method against tissue Doppler imaging. The statement "Strain imaging, however, is not widely used clinically in the assessment of diastolic impairment" expressing a disadvantage of the method is a counterargument for the main proposition. Cognitive actions are affected by means of a variety of markers. Lexical features are represented by professionalisms (tissue Doppler imaging, EF, speckle-tracking technique), terms (Myocardial strain assessment, heart, systolic and diastolic function). A high occurrence of medical terminology and medical professionalisms allows to present ideas accurately, and for this reason aids better understanding among peers. Persuasion is based on comparison of two techniques accomplished by intensifying markers (does provide, additional information), logical sequence markers (first, second, furthermore, therefore, however), comparison 
structure phrases (on the other hand, rather than). Modality aids to express uncertainty and probability by means of modals (can uncover, may not be apparent, may also be helpful) and modal modifiers (simply, erroneously, widely).

Medical professional discussions reveal lack of categorical judgments in presenting experts' claims. Participants usually begin their answer with I think... (I believe..., I suppose...) to show that this claim expresses his or her subjunctive position.

A.: What do you believe are the standards of care for heart failure patients for which this type of testing is desired or required?

B.: I think what's important to understand is that CPX testing has been a major component of research with heart failure patients...

Here the opening phrase I think seems to be polite and reasonable in this situation. Such phrases are considered as informal, so their usage indicates oral character of communication. It is possible to reinforce these phrases by means of specific markers, such as definitely (I definitely think that...), do with the verb (I do think that...) to express assurance of the speaker.

Recurrent use of the first-person pronoun $I$ indicates personal position of the speaker and aids to anticipate the danger of overstatement, while the first-person pronoun we indicates shared knowledge accepted by a professional group. Let us consider a fragment of a discussion:

A.: Dr. P., as an urologist, what do you think about the potential biases ...?

B.: I think that's a very fair criticism of the study... For example, we know for a fact that there are differences in the quality of surgical interventions between high-volume and low-volume hospitals. I suspect that the same differences exist for radiation therapy. Therefore, I think there are differences with regard to who underwent treatment and where the patients were treated in the European study.....

Here when the speaker presents his own opinion he usually uses the first-person pronoun and cognitive verbs $I$ think or $I$ suspect (to a lesser extent of assurance) to indicate subjectivity of the claim. Acting this way he leaves room for dialogue with his communicative partners in case they would have an alternative view.

Personal opinion may also be presented by means of phrases like my decisions about ..., my philosophy for ..., my take-home message ..., my practice ..., my personal opinion..., in my experience..., etc. They are less formal and appear in actual oral communication to express subjectivity of the viewpoint.

To build an argument in medical professional discussions are widely used specific markers which may be divided into the following groups: concession (however, nonetheless, although, meanwhile), reformulation (in other words, is that, generally, basically), doubt (perhaps, probably, potentially, most likely, actually), amplification (in addition, additionally, specifically), confirmation (certainly, in fact, really, understandably, extremely well).
Categorical assertions may be soften by means of modals when the expert summarizes his ideas. The speaker tends to avoid using modal verb must and prefers using have to or should to soften straightforwardness of the utterance.

- So, one has to be careful about using the maximal heart rate response to exercise testing as a reliable parameter of peak effort in clinical cardiology population.

- So, my take-home message is that we should not generalize the result of either study to all patients.

Here the synthesis of the experts' opinion sounds as a recommendation but not an obligation and in this sense is more preferred and polite.

Cognitive speech actions also include expressing agreement and disagreement with the previous statements or expressing positive or negative attitude to a discussed question. Expressing agreement with a partner's viewpoint is presented explicitly in examined discussions by phrases, such as I agree..., I completely agree..., I definitely agree.... However, expressing negation requires a speaker's additional effort to be polite and keep a positive atmosphere amongst peers. Implicit disagreement is more preferred in medical professional discussions. To criticize or contradict, the speaker should primarily agree and only then he may express disagreement. Let us consider the fragment of a discussion:

\section{A.: Do you pretty much agree with M.?}

B.: For the most part, yes. I tend to prefer RAS drugs as first step treatment and I don't think there is any difference in term of BP response between men and women.

Here there is a sequence of hedging to express the speaker's disagreement indirectly with the previous utterance. The speaker tends to understate negation to be polite. To express generally agreement at the beginning of the phrase he uses for the most part, yes, tend to prefer..., then according to cultural norms he rather uses not with the cognitive verb think than with the verb in the following clause [11].

Thus, cognitive speech actions serve for expressing an expert's opinion, building arguments, presenting agreement or disagreement with the communicative partner. It is conventionally accepted to soften categorical judgments and negation to develop communicative process in effective way.

\section{VIII.CONCLUSION}

In medical professional communication, specialists' communicative behavior is represented in the system of speech actions aimed at a successful informational exchange and production of novel professional knowledge. The analysis of medical professional discussion shows that the system is composed of connective, metacommunicative, directive, and cognitive speech actions. Connective speech actions aid to establish contact with all discussants and finish the communicative process maintaining positive atmosphere and attitude among peers. These speech actions are highly ritual and represented by means of special vocatives and speech etiquette formulae. Metacommunicative actions perform two functions: regulating communication and promoting understanding between participants. The main devices of these 
speech actions are modals, lexical markers of agreement, etiquette phrases, less informative particles. Directive speech actions serve to develop a discussion. They are primarily expressed by indirect requests, indirect questions, modal modifiers to meet English culture norms requiring minimization of the imposition on communicative partners. Cognitive speech actions are directed to sharing professional knowledge and expressing experts' viewpoint on actual medical issues. They are represented by means of modality devices, cognitive verbs, hedging, and specific markers.

\section{References}

[1] L.S. Beylinson, Professional discourse: features, functions, norms (on material of logopaedist's communicative practice), PhD Thesis, Volgograd, 2009, 339 p. (in Russian).

[2] V.V. Zhura, Discursive competence of a doctor in oral medical communication, Volgograd: VolgGMU, Dar-Co Publ., 2008. 376 p. (in Russian).

[3] N.Yu. Sidorova, Communicative behavior of unequal subjects in medical discourse, PhD Thesis, Volgograd, 2008, 177 p. (in Russian).

[4] E.G. Mishler, The Discourse of Medicine: Dialectics of Medical Interviews (Language and Learning for Human Service Professions). Norwood, N.J.: Ablex, 1984, 241 p.

[5] P. Atkinson, Medical Talk and Medical Work. London: Sage, 1995, $176 \mathrm{p}$.

[6] C.J. Pettinari, Task, talk and text in the operating room: A study in medical discourse. Norwood, NJ.: Ablex, 1988, 169 p.

[7] R.R. Anspach, "Notes on the Sociology of Medical Discourse: The Language of Case Presentation", Journal of Health and Social Behavior, vol. 29. pp. 357-375, 1988.
[8] I.A. Sterinin, Models of communicative behavior description, Voronezh: Garant Publ., 2015, 52 p. (in Russian).

[9] T.P. Skorikova, "Genre and functional typology and constructive and linguistic features of oral scientific-professional communication", Humanitarian Bulletin, vol. 3 No. 5, 2013. pp. 1-11. (in Russian).

[10] M.S. Nevzorova, "Communicative situation of oral professional communication within English-language medical discourse", Nauchnyy dialog, No. 3, pp. 68-80. (in Russian).

[11] T.V. Larina, Category of politeness and style of communication], Moscow: Rukopisnye pamyatniki Drevney Rusi, 2009, 516 p.

[12] N.L. Shamne, Actual problems of intercultural communication, Volgograd: Izd-vo VolGU Publ., 2014, 200 p.

[13] S. Borge, "Questions" in Pragmatics of speech actions, edit. M. Sbisa, K. Turner, Walter de Gruyter GmbH, Berlin, Boston, 2013, pp. 411-443.

[14] P. Webber, "Medical English: Conferencing" in Encyclopedia of Language and Lingiustics, 2nd ed., edit. K. Brown. Elsevier Science, 2006, pp. 721-724.

[15] K. Hyland, "Medical Discourse: Hedges" in Encyclopedia of Language and Lingiustics, 2nd ed, edit. K. Brown. Elsevier Science, 2006, pp. 694697.

[16] K. Hyland, English for Academic Purposes. London and New York, Routledge, 2006, $342 \mathrm{p}$.

[17] K. Kong, Professional discourse. Cambridge University Press. 2014, $300 \mathrm{p}$.

[18] D. Carnet, A. Magnet, "Medical Journals, Letters to the Editor" in Encyclopedia of Language and Lingiustics, 2nd ed., edit. K. Brown. Elsevier Science, 2006, pp. 725-732.

[19] R. Iedema, "Medicine and Health: Inter-and intra-professional Communication" in Encyclopedia of Language and Lingiustics, 2nd ed., edit. K. Brown. Elsevier Science, 2006, pp. 745-752. 